\title{
COMPUTING PRIME DIVISORS IN AN INTERVAL
}

\author{
MINKYU KIM AND JUNG HEE CHEON
}

\begin{abstract}
We address the problem of finding a nontrivial divisor of a composite integer when it has a prime divisor in an interval. We show that this problem can be solved in time of the square root of the interval length with a similar amount of storage, by presenting two algorithms; one is probabilistic and the other is its derandomized version.
\end{abstract}

\section{INTRODUCTION}

Integer factorization is one of the most challenging problems in computational number theory. It has been studied for centuries, and it has been intensively investigated after introducing the RSA cryptosystem 18 . The difficulty of integer factorization has been examined not only in pure factoring situations but also in various modified situations. One such approach is to find a nontrivial divisor of a composite integer when it has prime divisors of special form. These include Pollard's $p-1$ algorithm [15], Williams' $p+1$ algorithm [20, and others. On the other hand, owing to the importance and the usage of integer factorization in cryptography, one needs to examine this problem when some partial information about divisors is revealed. This side information might be exposed through the procedures of generating prime numbers or through some attacks against crypto devices or protocols.

This paper deals with integer factorization given the approximation of divisors, and it is motivated by the above mentioned research. More precisely, we are mainly interested in finding a nontrivial divisor of a composite integer $N$ when it has a divisor in an interval $[\alpha, \beta]$.

The declared problem can be solved in $\mathcal{O}(\beta-\alpha)$ time with trial division, while finding all the divisors in the interval. For sufficiently small interval length $\beta-\alpha$, all divisors in the interval can be recovered in polynomial time in $\log N$ using Coppersmith's method [5, 6] for finding small roots of modular polynomial equations. For relatively small $\alpha$ and large $\beta$, it is sufficient to use Pollard's algorithm [15, Theorem 1], with which one can find a nontrivial divisor in deterministic $\widetilde{\mathcal{O}}\left(\beta^{1 / 2+\gamma}\right)$ time for any $\gamma>0$, or to use Pollard's rho method [17, which runs in $\mathcal{O}\left(\beta^{1 / 2}\right)$ time. However, for large $\alpha$ and large $\beta-\alpha$, for example, when the most significant bits of a divisor are known, the exhaustive search combined with Coppersmith's method is the best known algorithm to find a divisor in an interval. Moreover, there is no known algorithm for finding a divisor in an interval with birthday complexity, that

Received by the editor December 15, 2012 and, in revised form, April 11, 2013 and May 10, 2013.

2010 Mathematics Subject Classification. Primary 11Y05, 11Y16; Secondary 68Q25.

Key words and phrases. Integer factorization, prime divisors in an interval.

This work was supported by the National Research Foundation of Korea (NRF) grant funded by the Korea Government (NRF-2011-0018345). 
is, the square root of the interval length, even though it is a natural requirement to design such an algorithm.

In this paper, we present two algorithms, one is probabilistic and the other is its deterministic version, for achieving birthday complexity in finding a divisor in an interval. The proposed algorithms can find a nontrivial divisor of a composite integer $N$ when it has a prime divisor in an interval, at around the time of the square root of the interval length with a similar amount of storage. As a result, using the proposed deterministic algorithm, we can check the existence of prime divisors in the interval, and if they exist, we can find all such prime divisors, by recursively applying the proposed deterministic algorithm at most $\log N$ times, in combination with a primality test such as [1] and a procedure to check that an integer belongs to the interval.

Our algorithms basically work by solving the discrete logarithm problem over $(\mathbb{Z} / n \mathbb{Z})^{*}$, where $n$ is an unknown divisor of the target composite integer $N$. To solve this problem efficiently, we adapt a multipoint evaluation method of univariate polynomials, as that of Pollard [15], who used it to give a deterministic time algorithm for finding divisors less than some integers. At the heart of the deterministic argument is the distribution of smooth numbers, that is, to take small integers to generate a large subgroup of $(\mathbb{Z} / N \mathbb{Z})^{*}$. We note that this approach was originally used for devising a deterministic primality test under some condition by Konyagin and Pomerance 9 .

Compared to previous algorithms to find a divisor in an interval, the proposed algorithms are more efficient for some parameters. The complexity of Coppersmith's method depends on not only the interval length but also the relative size of the divisor for the target composite number $N$. In contrast, the proposed algorithms mainly depend on the interval length. This difference leads to a situation where our algorithms are better than Coppersmith's method. As $\log N$ becomes larger or $\log (\beta-\alpha)$ gets closer to $\log \alpha$, our algorithms become more efficient than Coppersmith's method. For example, roughly speaking, when $\log N=4 \ell, \log \alpha=\ell$, $\log (\beta-\alpha)=2 \ell / 3$, the proposed algorithms run in $\mathcal{O}\left(2^{\ell / 3}\right)$ time , whereas Coppersmith's method runs in $\mathcal{O}\left(2^{5 \ell / 12}\right)$ time, which is less efficient.

Compared to Pollard's algorithm [15], the proposed algorithms are better for the case that $\beta-\alpha$ is significantly smaller than $\beta$. We also note that by combining our techniques in Section 5 and Pollard's technique in [15, Section 2] one can obtain another deterministic algorithm suitable to the interval case, but it is not as efficient as our solution. The detailed explanation can be found in Section 5.5. In fact, one may consider applying Pollard's kangaroo method [17] to find a factor in an interval. Pollard's kangaroo method solves the discrete logarithm problem by finding a collision between two sequences which are generated by iteratively applying a function $f$ to two distinct starting points. The main reason why this method works is that a collision between these two sequences is always preserved after applying $f$. However, this method as well as Pollard's rho method for the discrete logarithm [17] uses an iteration function $f$ which does not satisfy that $f(x \bmod p)=f(x) \bmod p$. Such an iteration function does not preserve a collision modulo $p$ after its applications, and so cannot be used for integer factorization.

The remainder of this paper is organized as follows. In Section 2 , we introduce notation and a theorem about smooth integers necessary for devising our deterministic algorithm. We start to present our algorithms by giving Lemma 3.1 in 
Section [3, which is frequently used in the paper. We also present Pollard's algorithm [15, Theorem 1] in Section 3. The proposed probabilistic algorithm is described in Section 4, and its derandomized version is given in Section 5. Our deterministic algorithm mainly consists of two subroutines, Algorithm 1 and Algorithm 2. First, we explain what can be done with these subroutines, and then, we describe our deterministic algorithm. The detailed explanation of these two subroutines is provided in the subsequent sections. In Section 6, we compare our algorithms mainly with Coppersmith's method. The final section summarizes the result of the present study and discusses further research.

\section{Preliminaries}

Let $a, b$ be integers. If $a$ divides $b$, this is denoted by $a \mid b$; otherwise, $a \nmid b$. We write $a^{\nu} \| b$ if $a^{\nu} \mid b$ and $a^{\nu+1} \nmid b$ for some integer $\nu$. In this case, we say $a^{\nu}$ exactly divides $b$. Let $\nu_{a}(b)$ denote the nonnegative integer such that $a^{\nu_{a}(b)} \| b$. Let $\varphi(\cdot)$ denote the Euler totient function and let $\omega(m)$ denote the number of distinct prime divisors of an integer $m$. Let $\operatorname{ord}_{m}(a)$ denote the order of $a$ modulo $m$, i.e., the smallest positive integer $n$ satisfying $a^{n} \equiv 1 \bmod m$. When using this notation, we will always assume that $\operatorname{gcd}(a, m)=1$. We will use "log" for the binary (base 2) logarithm and use "In" for the natural (base $e$ ) logarithm. We denote $[\alpha, \beta]$ as the set of all integers $\alpha \leq i \leq \beta$.

We say an integer $n$ is $y$-smooth if it is free of prime factors exceeding $y$. Let $\psi(x, y)$ denote the number of $y$-smooth integers $n \leq x$. The following theorem, proved in 9], gives a lower bound on $\psi(x, y)$, which will be used to prove our main results.

Theorem 2.1 (Konyagin and Pomerance). If $x \geq 4$ and $2 \leq y \leq x$,

$$
\psi(x, y)>x^{1-\ln \ln x / \ln y} .
$$

We use $\mathrm{M}_{\mathrm{p}}(d)$ to denote the number of operations in a ring $R$ for multiplying two polynomials in $R[X]$ of degree less than $d$, and we use the notation $\mathrm{M}(k)$ for the number of bit operations to perform a multiplication modulo an integer of $k$ bit size. As a result, the number of bit operations to multiply two polynomials of degree less than $d$ over $\mathbb{Z}_{m}$ is denoted by $\mathrm{M}_{\mathrm{p}}(d) \mathrm{M}(\log m)$. Note that from [19], we currently have

$$
\begin{aligned}
\mathrm{M}(k) & =\mathcal{O}(k \log k \log \log k)=\widetilde{\mathcal{O}}(k), \\
\mathrm{M}_{\mathrm{p}}(d) & =\mathcal{O}(d \log d \log \log d)=\widetilde{\mathcal{O}}(d),
\end{aligned}
$$

where $\widetilde{\mathcal{O}}(\ell)$ denotes $\mathcal{O}\left(\ell(\log \ell)^{\mathcal{O}(1)}\right)$.

\section{BASIC LEMma AND Pollard's Algorithm}

3.1. Basic lemma. Before describing our algorithms in the subsequent sections, we first give a lemma that is a slight modification of the lemma given in [15. Note that the following lemma relies on a classical algorithm to evaluate univariate polynomials at multiple points, and it will be frequently used in this paper.

Lemma 3.1. Given positive integers $N, g, h$, and $\delta$ with $1<g, h<N, \operatorname{gcd}(g h, N)=$ 1 , we can determine whether there is an integer $x \in[1, \delta]$ with

$$
\operatorname{gcd}\left(g^{x}-h, N\right)>1,
$$


and if so, find the least $x$ satisfying (3.1) in

$$
\mathcal{O}\left((\log \delta+\log \log N) \mathrm{M}_{\mathrm{p}}\left(\delta^{1 / 2}\right) \mathrm{M}(\log N)\right)
$$

bit operations by using storage for $\mathcal{O}\left(\delta^{1 / 2} \log \delta \log N\right)$ bits.

Proof. Let $L=\left\lceil\delta^{1 / 2}\right\rceil$. Since any integer $1 \leq x \leq \delta$ can be written as $x=j L-i$ for some integers $0 \leq i \leq L-1,1 \leq j \leq L$, we can check the existence of $x$ satisfying (3.1) by testing if there is some $i, j$ such that

$$
\operatorname{gcd}\left(g^{j L}-h g^{i}, N\right)>1 .
$$

A naive approach is to test (3.3) for all pairs $(i, j)$, which take $\mathcal{O}(\delta)$, but we want to do it more efficiently.

We first compute the polynomial

$$
F(X)=\prod_{0 \leq i \leq L-1}\left(X-h g^{i}\right) \bmod N .
$$

Then, we evaluate $F\left(g^{j L}\right) \bmod N$ and compute $d_{j}=\operatorname{gcd}\left(F\left(g^{j L}\right) \bmod N, N\right)$ for each $1 \leq j \leq L$. If there exists an integer $1 \leq j \leq L$ with $d_{j}>1$, we take the least such number, say $v$, and then, we find the greatest $u$ satisfying $\operatorname{gcd}\left(g^{v L}-h g^{u}, N\right)>$ 1. Note that such $u$ always exists since

$$
F\left(g^{v L}\right) \equiv \prod_{0 \leq i \leq L-1}\left(g^{v L}-h g^{i}\right) \equiv 0 \bmod p
$$

for some prime divisor $p$ of $d_{v}$. Thus, we have the least solution $x:=v L-u$ satisfying (3.1). Otherwise, if there is no such $j$ satisfying $d_{j}>1$, we conclude that there is no $x \in[1, \delta]$ satisfying (3.1).

It is a classical result [7, Section 10] that all the coefficients of $F$ can be computed with $\mathcal{O}\left(\log L \mathrm{M}_{\mathrm{p}}(L)\right)$ operations in $\mathbb{Z} / N \mathbb{Z}$ and the polynomial $F$ can be evaluated at multiple points $g^{j L}$ for all $1 \leq j \leq L$ with $\mathcal{O}\left(\log L \mathrm{M}_{\mathrm{p}}(L)\right)$ operations in $\mathbb{Z} / N \mathbb{Z}$, using storage for $\mathcal{O}(L \log L)$ elements in $\mathbb{Z} / N \mathbb{Z}$, which is at most $\mathcal{O}(L \log L \log N)$ bits. In addition, we perform GCD computation at most $2 L$ times, requiring $\mathcal{O}(L \log \log N \mathrm{M}(\log N))$ bit operations [7, Section 11]. Since $L \leq \mathrm{M}_{\mathrm{p}}(L)$, the overall complexity becomes $\mathcal{O}\left((\log L+\log \log N) \mathrm{M}_{\mathrm{p}}(L) \mathrm{M}(\log N)\right)$ bit operations.

Using FFT-based multiplication algorithms, (3.2) becomes $\widetilde{\mathcal{O}}\left(\delta^{1 / 2} \log N\right)$ bit operations. Note that this is the typical situation we have in mind while applying Lemma 3.1

3.2. Pollard's algorithm. We note that Pollard's algorithm will be used as a subroutine of our new deterministic algorithm. For easy reference and a self-contained paper, we present Pollard's algorithm [15] and its analysis. Pollard's algorithm always computes a nontrivial divisor of an integer $N$, provided that it has a prime divisor less than or equal to $\beta$. Let $\gamma$ be a positive real number. Pollard's algorithm works as follows:

(1) For $2 \leq a \leq A:=\left\lceil\beta^{\gamma}\right\rceil$, do

(1.a) If $\operatorname{gcd}(a, N)>1$, then output this and stop.

(1.b) Compute the least integer $x_{a} \in[1, \beta-\alpha]$ satisfying (3.1) with $g=a$, $h=1$, by using the technique described in Lemma 3.1

- If $\operatorname{gcd}\left(a^{x_{a}}-1, N\right)<N$, then output this and stop. 
- If there is no such $x_{a}$, then state that $N$ has no prime divisor $\leq \beta$, and stop.

(2) Let $H$ be the least common multiple of $x_{2}, \ldots, x_{A}$.

- If $z H+1$ divides $N$ for some integer $1 \leq z \leq C(1 / \gamma)^{1 / \gamma}$, output this and stop; otherwise, state that $N$ has no prime divisor $\leq \beta$, and stop.

In the above description, $C$ is the number defined in the following lemma, which is essentially proved in [15, Section 2].

Lemma 3.2. Let $p$ be a prime and $u>1$. There is a constant $C>0$ such that, given a prime $p$, the minimal $z$-th power nonresidue $\bmod p$ is less than $p^{1 / u}$ for any divisor $z$ of $p-1$ with $z>C u^{u}$.

Proof. We know that $\psi\left(x, x^{1 / u}\right)=\rho(u) x+\mathcal{O}(x / \log x)$ for a Dickman function $\rho(u)=u^{-\mathcal{O}(u)}$. Thus, there is a constant $C>0$ such that $\psi\left(x, x^{1 / u}\right) \geq \frac{x}{C x^{x}}$ for any integer $x$. Suppose that there exists a divisor $q$ of $p-1$ with $q>C u^{u}$ such that all integers up to $p^{1 / u}$ are $q$-th power residues $\bmod p$. Since any product of $q$-th power residue is also a $q$-th power residue, we have at least $\phi\left(p, p^{1 / u}\right) q$-th power residues in $[1, p-1]$. However, it is impossible because $\phi\left(p, p^{1 / u}\right) \geq p /\left(C u^{u}\right)$ is larger than $(p-1) / q$, the number of all $q$-th power residues in $[1, p-1]$.

3.2.1. Analysis. Suppose that there is a prime divisor $p$ of $N$ with $p \leq \beta$. Then for any integer $a$ relatively prime to $N$ there is certainly the integer $x_{a}$ in Step (1.b), since $a^{p-1} \equiv 1 \bmod p$ by Fermat's little theorem. If $\operatorname{gcd}\left(a^{x_{a}}-1, N\right)$ is less than $N$, we find a nontrivial divisor of $N$.

We now assume that $\operatorname{gcd}(a, N)=1$ and $\operatorname{gcd}\left(a^{x_{a}}-1, N\right)=N$ for any integer $2 \leq a \leq A$. Let $H=\operatorname{lcm}\left(x_{2}, \ldots, x_{A}\right)$. We claim that any prime divisor $p$ of $N$ with $p \leq \beta$ is of the form $p=z H+1$ for some integer $z \in\left[1, C u^{u}\right]$, where $u=1 / \gamma$. Since $a^{x_{a}} \equiv 1 \bmod p$ and $x_{a}$ is minimal with this property, we have $x_{a} \mid p-1$ for each $a$ and so $H \mid p-1$. Suppose that there is a prime divisor $p$ of $N$ with $z:=(p-1) / H>C u^{u}$. Then, by Lemma 3.2, there exists a $z$-th power nonresidue $\bmod p$, say $b$, less than $A$. Since $b$ is $z$-th power nonresidue $\bmod p$, we have $b^{(p-1) / z} \not \equiv 1 \bmod p$, which contradicts the fact that $H=(p-1) / z$ is a multiple of $x_{b}=\operatorname{ord}_{p}(b)$. Thus, if $\operatorname{gcd}\left(a^{x_{a}}-1, N\right)=N$ for any $2 \leq a \leq A$, the described algorithm outputs a nontrivial divisor of $N$ in Step (2).

The overall complexity of Pollard's algorithm is

$$
\mathcal{O}\left(\beta^{\gamma}(\log \beta+\log \log N) \mathrm{M}_{\mathrm{p}}\left(\beta^{1 / 2}\right) \mathrm{M}(\log N)\right)
$$

bit operations by using the storage for $\mathcal{O}\left(\beta^{1 / 2} \log \beta \log N\right)$ bits.

\section{Probabilistic Algorithm}

In this section, we present our probabilistic algorithm for computing a nontrivial divisor of a composite integer $N$, provided that the target integer $N$ has a prime divisor $p$ in the interval $[\alpha, \beta]$. In the remainder of this paper, we will always assume that $N$ is an odd integer, and not a perfect power. Note that we can efficiently factor $N$ if $N \geq 2$ is a perfect power, i.e., if $N=n^{k}$ for some integers $n \geq 2, k \geq 2$ [14, Section 3.6]. 
4.1. Algorithm description and complexity. Let us take a positive integer $a$, which is relatively prime to $N$, and let $h_{a} \equiv a^{\beta-1} \bmod N$. Let $p$ denote an unknown prime divisor of $N$. Since $a^{p-1} \equiv 1 \bmod p$ by Fermat's little theorem and $\beta-1-(p-1)=\beta-p \in[0, \beta-\alpha]$, if $\beta \nmid N$, there is an integer $x_{a} \in[1, \beta-\alpha]$ satisfying $d:=\operatorname{gcd}\left(a^{x_{a}}-h_{a}, N\right)>1$, and such an integer can be computed by applying the technique described in Lemma 3.1 to $g=a, h=h_{a}$, and $\delta=\beta-\alpha$. If $d<N$, a nontrivial divisor of $N$ is found. If $d=N$ and $y_{a}$ is even, we also hope to find a nontrivial divisor of $N$ by computing $\operatorname{gcd}\left(a^{y_{a} / 2^{i}}-1, N\right)$ for $i=1, \ldots, \nu_{2}\left(y_{a}\right)$, where $y_{a}=\beta-1-x_{a}$. This is summarized in the following:

(1) Choose an integer $a$ uniformly at random in $\{2, \ldots, N-1\}$.

- If $\operatorname{gcd}(a, N)>1$, then output this and stop.

(2) Compute $x_{a} \in[1, \beta-\alpha]$ such that $d=\operatorname{gcd}\left(a^{x_{a}}-a^{\beta-1} \bmod N, N\right)>1$ by applying the technique described in Lemma 3.1

- If $d<N$, then output this and stop.

- If there is no such $x_{a}$, then state that $N$ has no prime divisor in the interval $[\alpha, \beta]$ and stop.

(3) If $y_{a}:=\beta-1-x_{a}$ is even, then compute $d_{i}=\operatorname{gcd}\left(a^{y_{a} / 2^{i}}-1, N\right)$ for $i=1, \ldots, \nu_{2}\left(y_{a}\right)$.

- If $1<d_{i}<N$ for some $1 \leq i \leq \nu_{2}\left(y_{a}\right)$, output this and stop.

(4) Output "failure" and stop.

Since Step (3) can be performed with one exponentiation in $(\mathbb{Z} / N \mathbb{Z})^{*}$ by an exponent less than $\beta$ and $\nu_{2}\left(y_{a}\right)$ GCD computations, the proposed probabilistic algorithm takes

$$
\mathcal{O}\left((\log \delta+\log \log N) \mathrm{M}_{\mathbf{p}}\left(\delta^{1 / 2}\right) \mathrm{M}(\log N)+\log \beta \log \log N \mathrm{M}(\log N)\right)
$$

bit operations. Under the conditions $\log \beta \leq \delta^{1 / 2}$ and $\log N \leq \delta$, this is equal to

$$
\mathcal{O}\left(\log \delta \mathrm{M}_{\mathrm{p}}\left(\delta^{1 / 2}\right) \mathrm{M}(\log N)\right)
$$

bit operations. The storage requirement is $\mathcal{O}\left(\delta^{1 / 2} \log \delta \log N\right)$ bits.

4.2. Success probability. The starting point to analyze success probability is the following theorem [2, Section 3].

Theorem 4.1 (Bach [2]). Let $N$ be an odd positive integer, not a prime power and let its complete factorization be $N=\prod_{i=1}^{\ell} q_{i}^{e_{i}}$. Let $\lambda=\operatorname{lcm}\left(\varphi\left(q_{1}^{e_{1}}\right), \ldots, \varphi\left(q_{\ell}^{e_{\ell}}\right)\right)$. We consider the subset $\mathcal{K}$ of $(\mathbb{Z} / N \mathbb{Z})^{*}$ :

$$
\mathcal{K}=\left\{1 \leq a<N: \operatorname{gcd}(a, N)=1 \text { and } a^{\lambda / 2} \equiv \pm 1 \bmod N\right\} .
$$

Then, we have the following:

- $\mathcal{K}$ is a proper subgroup of $(\mathbb{Z} / N \mathbb{Z})^{*}$, and hence, $|\mathcal{K}| \leq \frac{\varphi(N)}{2}$.

- For any $a \in(\mathbb{Z} / N \mathbb{Z})^{*} \backslash \mathcal{K}$ and $y \in \mathbb{Z}$ such that $a^{y} \equiv 1 \bmod N$, we must have $\nu_{2}(y) \geq 1$ and $a^{y / 2^{k}} \equiv 1 \bmod N$, but $a^{y / 2^{k+1}} \not \equiv \pm 1 \bmod N$ for some $0 \leq k<\nu_{2}(y)$.

Based on this theorem, we can compute the success probability of our probabilistic algorithm as follows. Let $a$ be a positive integer that fails to find a nontrivial divisor of $N$ by applying Lemma 3.1. If $a$ is chosen uniformly at random from the set $\{1 \leq b \leq N-1: \operatorname{gcd}(b, N)=1\}$, by Theorem 4.1, with probability of at least $1 / 2$, we have that $a \notin \mathcal{K}$, and so $y_{a}$ is even and $1<\operatorname{gcd}\left(a^{y_{a} / 2^{k+1}} \pm 1, N\right)<N$ for some 
$0 \leq k<\nu_{2}\left(y_{a}\right)$. Thus, if $a \notin \mathcal{K}$, then one of $\operatorname{gcd}\left(a^{y_{a} / 2^{i}}-1, N\right)$ for $i=1, \ldots, \nu_{2}\left(y_{a}\right)$ must be a nontrivial divisor of $N$. This shows that our algorithm succeeds with a probability of at least $1 / 2$.

\section{Deterministic Algorithm}

The drawback of the algorithm described in the previous section is that we do not know exactly how many $a$ 's are to be tested or how to choose $a$ to split $N$, and hence, the algorithm works probabilistically. However, under the Extended Riemann Hypothesis (ERH), we can actually prove the existence of a positive integer $a \leq 2 \ln ^{2} N$ such that one of $\operatorname{gcd}(a, N), \operatorname{gcd}\left(a^{y_{a}}-1, N\right), \operatorname{gcd}\left(a^{y_{a} / 2}-1, N\right)$, $\ldots, \operatorname{gcd}\left(a^{y_{a} / 2^{\nu_{2}\left(y_{a}\right)}}-1, N\right)$ gives a nontrivial divisor of $N$ by applying the following theorem to $\mathbb{G}=\mathcal{K}$.

Theorem 5.1 (Bach 3). Assuming the ERH is true, for any positive integer $n$ and any proper subgroup $\mathbb{G} \varsubsetneqq(\mathbb{Z} / n \mathbb{Z})^{*}$, the least positive integer a such that $a \in(\mathbb{Z} / n \mathbb{Z}) \backslash \mathbb{G}$ is at most $2 \ln ^{2}(n)$, and the least positive integer $b$ such that $b \in$ $(\mathbb{Z} / n \mathbb{Z})^{*} \backslash \mathbb{G}$ is at most $3 \ln ^{3}(n)$.

The question is whether one can give another deterministic algorithm without unproven assumptions as in the ERH, while maintaining the complexity at the same level as the probabilistic algorithm. In this section, we answer this question successfully.

5.1. Algorithm description. To derandomize our probabilistic algorithm, we will give additional strategies to find a nontrivial divisor when we have $\operatorname{gcd}\left(a^{y_{a}}-1, N\right)=$ $N$ for many integers $a$. We first present these procedures, and then state our deterministic algorithm. The detailed analysis of our algorithm is given in the subsequent section.

As a first step, we extend the final process in the probabilistic algorithm to enhance the possibility of finding a nontrivial divisor of $N$. That is, one might be able to find a nontrivial divisor of $N$ by computing $\operatorname{gcd}\left(a^{y_{a} / s^{i}}-1, N\right), 1 \leq i \leq$ $\nu_{s}\left(y_{a}\right)$, for prime divisors $s$ of $y_{a}$. In fact, this is a process for checking whether there is a prime divisor $s$ of $\operatorname{ord}_{N}(a)$ with $1<\operatorname{gcd}\left(a^{\operatorname{ord}_{N}(a) / s_{-}}-1, N\right)<N$, which is equivalent to the existence of a nontrivial divisor $n$ of $N$ satisfying $\operatorname{ord}_{N}(a) \neq \operatorname{ord}_{n}(a)$. The obstacle to apply this approach is that the prime divisors of $y_{a}$ are unknown and the size of $y_{a}$ is almost $\beta$, but we want to keep the complexity at the level of $(\beta-\alpha)^{1 / 2}$. In the following lemma, we show how to overcome this problem.

Lemma 5.2. Let $N$ be a composite integer that has a prime divisor $p$ in the interval $[\alpha, \beta], \delta=\beta-\alpha$, and let $a$ be an integer with $\operatorname{gcd}(a, N)=1$. Given an integer $y \in[\alpha-1, \beta-1]$ satisfying $a^{y} \equiv 1 \bmod N$, for any $\gamma>0$, we can compute a nontrivial divisor of $N$ or the order of a modulo $N$ including its complete factorization in

$$
\mathcal{O}\left(\delta^{\gamma} \log \beta(\log \delta+\log \log \beta) \mathrm{M}_{\mathbf{p}}\left(\delta^{1 / 2}\right) \mathrm{M}(\log \beta)+\log \beta(\log \beta+\log \log N) \mathrm{M}(\log N)\right)
$$

bit operations by using storage for $\mathcal{O}\left(\delta^{1 / 2} \log \delta \log \beta\right)$ bits, where $\delta=\beta-\alpha$. Moreover, whenever we fail to obtain a nontrivial divisor of $N$, we have that $\operatorname{ord}_{N}(a)=$ $\operatorname{ord}_{n}(a)$ for any divisor $n$ of $N$.

Proof. See Section 5.3 . 
In the next step, we try to find a nontrivial divisor of $N$ using the information gathered by applying Lemma 5.2 to many integers $a$. Note that if we fail to find a nontrivial divisor by applying Lemma 5.2 to an integer $a$, we obtain the order of $a$ modulo $N$ and its complete factorization. At first glance, this information does not seem useful because we also know that $\operatorname{ord}_{N}(a)$ is equal to $\operatorname{ord}_{n}(a)$ for all divisors $n$ of $N$. However, gathering such integers and comparing their orders, we can go further to find a nontrivial divisor of $N$.

To explain this method more precisely, let us consider the situation where we have two different integers $a$ and $b$ along with their order modulo $N$. If $d:=$ $\operatorname{gcd}\left(\operatorname{ord}_{N}(a), \operatorname{ord}_{N}(b)\right)$ is greater than 1, we still have another chance to find a nontrivial divisor of $N$. This is because two integers $g:=a^{\operatorname{ord}_{N}(a) / d} \bmod N$ and $h:=b^{\operatorname{ord}_{N}(b) / d} \bmod N$ have the same order $d$ modulo $N$, which implies that there is an integer $x \in[1, d]$ such that $d^{\prime}=\operatorname{gcd}\left(g^{x}-h, N\right)>1$. Thus, by applying Lemma 3.1, we may be able to find a nontrivial divisor of $N$. The problem is that when $d>\beta-\alpha$ this approach requires more computation than $(\beta-\alpha)^{1 / 2}$, which is an undesirable result. However, this problem can be overcome by applying the Pohlig-Hellman technique because the typical situation we encounter is that $\operatorname{ord}_{N}(a)$ and $\operatorname{ord}_{N}(b)$ are $(\beta-\alpha)$-smooth and their complete factorizations are known. This is summarized in the following lemma.

Lemma 5.3. Let $a, b$ be two distinct integers that are relatively prime to $N$. Assume that $\operatorname{ord}_{N}(a), \operatorname{ord}_{N}(b)$, and their complete factorizations are known. We can find either a nontrivial divisor of $N$ or a generator of the subgroup of $(\mathbb{Z} / N \mathbb{Z})^{*}$ generated by $a$ and $b$ in

$$
\mathcal{O}\left(\log d \log \log N \mathrm{M}_{\mathrm{p}}\left(s^{1 / 2}\right) \mathrm{M}(\log N)+\log ^{2} d \mathrm{M}(\log N)\right)
$$

bit operations by using storage for $\mathcal{O}\left(s^{1 / 2} \log s \log N\right)$ bits, where $s$ is the largest prime divisor of $d=\operatorname{gcd}\left(\operatorname{ord}_{N}(a), \operatorname{ord}_{N}(b)\right)$.

Proof. See Section 5.4.

Our new deterministic algorithm works by repeatedly applying the two steps and Lemma 3.1 to the integers in the interval $\left[2,(\ln N)^{1 /(1-\epsilon)}\right]$, where $\epsilon$ denotes a positive real number with $N^{\epsilon}=\min \left\{\beta, N^{1 / 2}\right\}$. The detailed procedure is described in the following:

(I) For $a=2$ to $A:=\left\lfloor(\ln N)^{1 /(1-\epsilon)}\right\rfloor$, do

(1) Check if $a$ is relatively prime to $N$.

- If $\operatorname{gcd}(a, N)>1$, then output this and stop.

(2) Compute $x_{a} \in[1, \beta-\alpha]$ such that $d=\operatorname{gcd}\left(a^{x_{a}}-a^{\beta-1} \bmod N, N\right)>1$ by applying the technique described in Lemma 3.1

- If $d<N$, then output this and stop.

- If there is no such $x_{a}$, then state that $N$ has no prime divisor in the interval $[\alpha, \beta]$, and stop.

(3) Apply the algorithm associated with Lemma 5.2, called Algorithm 1, with inputs $a$ and $y_{a}=\beta-1-x_{a}$. (Algorithm 1 is described in Section 5.3)

- If a nontrivial divisor of $N$ is found, then output this and stop.

- Otherwise, Algorithm 1 outputs $\operatorname{ord}_{N}(a)$ including its complete factorization.

(4) If $a=2$, set $b=a$; otherwise, do 
(4.a) Apply the algorithm associated with Lemma 5.3, called Algorithm 2, with inputs $a, b$, and complete factorizations of $\operatorname{ord}_{N}(a), \operatorname{ord}_{N}(b)$. (Algorithm 2 is described in Section 5.4)

- If a nontrivial divisor of $N$ is found, then output this and stop.

- Otherwise, Algorithm 2 outputs a generator $\zeta$ of the subgroup generated by $a$ and $b$, and $\operatorname{ord}_{N}(\zeta)$ including its complete factorization.

(4.b) Set $b=\zeta$.

(II) State that $N$ has no prime divisor in the interval $[\alpha, \beta]$.

5.2. Analysis. We claim that the described algorithm always terminates by giving a nontrivial divisor of $N$. We note that the main idea for the deterministic argument was originally used for devising a deterministic primality test under some condition by Konyagin and Pomerance 9 .

Assume that we fail to find a nontrivial divisor of $N$ by applying Step (1), Step (2), and Step (3) to all $2 \leq a \leq A$. In this case, we know $\operatorname{ord}_{N}(a)$ including its complete factorization and $\operatorname{ord}_{N}(a)=\operatorname{ord}_{n}(a)$ for any divisor $n$ of $N$. To prove the claim, it is sufficient to show that the subgroup $\mathbb{G}$ of $(\mathbb{Z} / N \mathbb{Z})^{*}$ generated by the set $S=\{2, \ldots, A\}$ is not cyclic. This is because, if $\mathbb{G}$ is not cyclic, we encounter two integers $a$ and $b$ in Step (4) such that the subgroup generated by $a$ and $b$ is not generated by a single element, and thus the algorithm has to terminate with the first bullet point of Step (4.a).

It now remains to show that $\mathbb{G}$ is not cyclic. If $\mathbb{G}$ is cyclic, then $|\mathbb{G}|$ is equal to the least common multiple of $\operatorname{ord}_{N}(2), \ldots, \operatorname{ord}_{N}(A)$. We also know that $|\mathbb{G}|$ is a divisor of $q-1$ for any prime divisor $q$ of $N$ because $\operatorname{ord}_{N}(a)=\operatorname{ord}_{q}(a)$ and $\operatorname{ord}_{q}(a)$ divides $q-1$. Thus, taking $q$ as the prime divisor of $N$ less than or equal to $N^{\epsilon}=\min \left\{\beta, N^{1 / 2}\right\}$, we have

$$
|\mathbb{G}|<q \leq N^{\epsilon}
$$

On the other hand, since

$$
\psi\left(N,(\ln N)^{1 /(1-\epsilon)}\right)>N^{\epsilon}
$$

by Theorem 2.1, and $\mathbb{G}$ is generated by $2, \ldots, A=\left\lfloor(\ln N)^{1 /(1-\epsilon)}\right\rfloor$, the subgroup $\mathbb{G}$ has more than $N^{\epsilon}$ elements. Thus, there is a contradiction.

Finally, referring to Lemma 3.1, Lemma 5.2, Lemma 5.3, and the fact that $\operatorname{ord}_{N}(a)$ is $\delta$-smooth, $\operatorname{gcd}\left(\operatorname{ord}_{N}(a), \operatorname{ord}_{N}(b)\right) \leq N$, and $A \leq \log ^{2} N$, we obtain the following theorem.

Theorem 5.4. If a composite integer $N$ has a prime divisor in the interval $[\alpha, \beta]$, for any $\gamma>0$, we can find a nontrivial divisor of $N$ in

$$
\mathcal{O}\left(\delta^{\gamma} \log ^{4} N \mathrm{M}_{\mathrm{p}}\left(\delta^{1 / 2}\right) \mathrm{M}(\log N)\right)
$$

bit operations by using storage for $\mathcal{O}\left(\delta^{1 / 2} \log \delta \log N\right)$ bits, where $\delta$ denotes $\beta-\alpha$.

In the following two subsections, we present the proof of Lemmas 5.2 and 5.3 . 
5.3. Proof of Lemma 5.2. First, suppose that $\operatorname{ord}_{N}(a)$ has a prime divisor $s>\delta$. In this case, we can always find a nontrivial divisor of $N$ as follows. Noting that $\operatorname{ord}_{N}(a) \mid \varphi(N)$, we know that $s \mid N$ or $s \mid q-1$ for some prime $q \mid N$. If $s$ is a divisor of $N$, then $\operatorname{gcd}(y, N)$ gives a nontrivial divisor of $N$. Now, we assume that $s \mid q-1$ for some prime $q \mid N$. If $s$ is a divisor of $q-1$ for some prime divisor $q \in[\alpha, \beta]$ of $N, y+1$ becomes a nontrivial divisor of $N$ because there is only one multiple of $s$ in the interval $[\alpha-1, \beta-1]$, and both $y$ and $q-1$ are multiples of $s$ in this interval.

It remains to consider the case that all the prime divisors $s>\delta$ of $\operatorname{ord}_{N}(a)$ are divisors of $q-1$ for some prime divisor $q \notin[\alpha, \beta]$ of $N$, and there is at least one such $s$. Since such integers $s$ do not divide $p-1$ and $\operatorname{ord}_{p}(a) \mid p-1$, we have that $\operatorname{ord}_{p}(a) \neq \operatorname{ord}_{N}(a)$ and $\operatorname{ord}_{p}(a)$ is $\delta$-smooth. This implies that $a^{m} \equiv 1 \bmod p$, but $a^{m} \not \equiv 1 \bmod N$, where $m$ is the largest $\delta$-smooth divisor of $y$. Thus, $\operatorname{gcd}\left(a^{m}-1, N\right)$ becomes a nontrivial divisor of $N$.

Second, suppose that all the prime divisors of $\operatorname{ord}_{N}(a)$ are at most $\delta$. Let $m$ be the integer defined as above. We can compute $m$ including its complete factorization by recursively applying Pollard's algorithm [15, theorem 1] at most $\log y$ times; the detailed procedure is presented below (Step (3)).

Let $s$ be a prime divisor of $m$ and let $e_{s}$ be the least nonnegative integer such that $\operatorname{gcd}\left(a^{m s^{e_{s}-\nu_{s}(m)}}-1, N\right)>1$. If $d_{s}:=\operatorname{gcd}\left(a^{m s^{e_{s}-\nu_{s}(m)}}-1, N\right)<N$, a nontrivial divisor of $N$ is found; otherwise, we have $s^{e_{s}} \| \operatorname{ord}_{n}(a)$ for any divisor $n$ of $N$. Thus, if $d_{s}=N$ for all prime divisors $s$ of $m$, we have $\operatorname{ord}_{N}(a)=\prod_{\text {prime } s \mid m} s^{e_{s}}$ and $\operatorname{ord}_{N}(a)=\operatorname{ord}_{n}(a)$ for any divisor $n$ of $N$.

The overall procedure, denoted as Algorithm 1, is summarized in the following:

Given inputs $N, a$, and $y$ such that $a^{y} \equiv 1 \bmod N$, do

(1) Compute $\operatorname{gcd}(y, N)$.

- If it gives a nontrivial divisor of $N$, then output this and stop.

(2) Check if $y+1$ divides $N$.

- If so, then output this and stop.

(3) Compute the largest $\delta$-smooth divisor $m$ of $y$ including its complete factorization as follows:

(3.a) Compute two nontrivial divisors $d_{1}, d_{2}=N / d_{1}$ by applying Pollard's algorithm described in Section 3.2 on inputs $(y, \delta)$, where the first argument $y$ denotes the integer which we want to factor, and the second argument $\delta$ denotes an upper bound of its prime divisors.

(3.b) Test if $d_{1}, d_{2} \leq \delta$.

(i) For $d_{i} \leq \delta$, apply Pollard's algorithm on inputs $\left(d_{i}, d_{i}^{1 / 2}\right)$.

- If it fails to factor $d_{i}$, output this as a prime divisor $\leq \delta$ of $n$.

- Otherwise, recursively apply this step to two divisors of $d_{i}$, respectively.

(ii) For $d_{j}>\delta$, apply Pollard's algorithm on inputs $\left(d_{j}, \delta\right)$.

- If it fails to factor $d_{j}$, stop to find a prime divisor $\leq \delta$ of $n$ using $d_{j}$.

- Otherwise, recursively apply Step (3.b) to two divisors of $d_{j}$, respectively.

(3.c) Gathering all prime divisors obtained in the first bullet point of Step (3.b)(i), we can find $m$ and its complete factorization. 
(4) For each prime divisor $s$ of $m$, compute the least nonnegative integer $e_{s}$ satisfying $\operatorname{gcd}\left(a^{m s^{e_{s}-\nu_{s}(m)}}-1, N\right)>1$.

- If one of these greatest common divisors is less than $N$, then output this and stop.

- Otherwise, output $\operatorname{ord}_{N}(a)=\prod_{\text {prime } s \mid m} s^{e_{s}}$.

Since a GCD computation takes $\mathcal{O}(\log \log N \mathrm{M}(\log N))$ bit operations and an exponentiation in $\mathbb{Z} / N \mathbb{Z}$ by an exponent less than $m$ requires $\mathcal{O}(\log m \mathrm{M}(\log N))$ bit operations, Step (1), Step (2), and Step (4) can be done in

$$
\mathcal{O}(\log m(\log m+\log \log N) \mathrm{M}(\log N))
$$

bit operations. In addition, since we apply Pollard's algorithm at most $\log y$ times in Step (3), for any $\gamma>0$, the number $m$ can be computed in

$$
\mathcal{O}\left(\delta^{\gamma} \log y(\log \delta+\log \log y) \mathrm{M}_{\mathrm{p}}\left(\delta^{1 / 2}\right) \mathrm{M}(\log y)\right)
$$

bit operations. Finally, combining (5.2), (5.3), and $m \leq y \leq \beta$, we achieve the claimed complexity.

5.4. Proof of Lemma 5.3, Before going to the proof of Lemma 5.3, we present a technical lemma. We note that the original idea of Lemma 5.5] and Lemma 5.3 is adopted from [9] as the deterministic argument in Section 5.2 .

Lemma 5.5. Let $a, b$ be two integers with

$$
\operatorname{ord}_{N}(a)=s^{e} \quad \text { and } \quad b^{s^{e}} \equiv 1 \bmod N,
$$

where $s$ is a prime number and $e \geq 1$. We can find either a nontrivial divisor of $N$ or an integer $x \in\left[0, s^{e}\right)$ satisfying $b \equiv a^{x} \bmod N$ in

$$
\mathcal{O}\left(e(\log s+\log \log N) \mathrm{M}_{\mathrm{p}}\left(s^{1 / 2}\right) \mathrm{M}(\log N)+e^{2} \log s \mathrm{M}(\log N)\right)
$$

bit operations by using storage for $\mathcal{O}\left(s^{1 / 2} \log s \log N\right)$ bits.

Proof. Before starting the proof, let us consider the situation where an integer $g$ has $\operatorname{ord}_{N}(g)=s$ and an integer $h$ satisfies $h^{s} \equiv 1 \bmod N$. Since $s$ is prime, there is a prime divisor $p$ of $N$ so that $\operatorname{ord}_{p}(g)=s$. The fact that $h^{s} \equiv 1 \bmod p$ and $\mathbb{Z}_{p}^{\times}$ is cyclic guarantees the existence of $x \in[0, s)$ such that $h \equiv g^{x} \bmod p$, and such $x$ can be computed by applying the technique in Lemma 3.1. If $\operatorname{gcd}\left(h-g^{x}, N\right)<N$, we have a nontrivial divisor of $N$. Otherwise, we have $h \equiv g^{x} \bmod N$.

Now, we continue to prove the lemma. Let $g=a^{s^{e-1}} \bmod N$ and $h_{0}=b^{s^{e-1}} \bmod$ $N$. It is clear that $\operatorname{ord}_{N}(g)=s$ and $h_{0}^{s} \equiv 1 \bmod N$. Thus, by applying the method described above, we can find an integer $x_{0} \in[0, s)$ such that $d_{0}:=\operatorname{gcd}\left(h_{0}-\right.$ $\left.g^{x_{0}}, N\right)>1$. If $d_{0}<N$, then $d_{0}$ is a nontrivial divisor of $N$. If $d_{0}=N$, we have $h_{0} \equiv g^{x_{0}} \bmod N$, and we compute $h_{1}=\left(b a^{-x_{0}}\right)^{s^{e-2}} \bmod N$. Since $h_{1}^{s} \equiv 1 \bmod N$, the situation is the same as before; find an integer $x_{1} \in[0, s)$ such that $d_{1}:=$ $\operatorname{gcd}\left(h_{1}-g^{x_{1}}, N\right)>1$, and if $d_{1}<N$, we have a nontrivial divisor. In general, for each $1 \leq i \leq e-1$, if we fail to find a nontrivial divisor in the previous procedure, we encounter the following situations: $d_{j}:=\operatorname{gcd}\left(h_{j}-g^{x_{j}}, N\right)=N$ for all $0 \leq j<i$ and $h_{i}=\left(b a^{-\left(x_{0}+\cdots+x_{i-1} s^{i-1}\right)}\right)^{s^{e-(i+1)}} \bmod N$. Eventually, if $d_{i}=\operatorname{gcd}\left(h_{i}-g^{x_{i}}, N\right)=N$ for all $0 \leq i \leq e-1$, we have $b a^{-\left(x_{0}+\cdots+x_{e-2} s^{e-2}\right)} \equiv a^{x_{e-1} s^{e-1}} \bmod N$, and hence, we obtain the integer $x=x_{0}+x_{1} s+\cdots+x_{e-1} s^{e-1}$ such that $b \equiv a^{x} \bmod N$. 
Finally, since the technique in Lemma 3.1, exponentiation in $(\mathbb{Z} / N \mathbb{Z})^{*}$ by an exponent less than $s^{e}$, and GCD computation are done $\mathcal{O}(e)$ times, we reach our conclusion.

Proof of Lemma 5.3 . Let $G$ be the subgroup of $(\mathbb{Z} / N \mathbb{Z})^{*}$ generated by $a$ and $b$, and let $x_{a}, x_{b}$ denote $\operatorname{ord}_{N}(a), \operatorname{ord}_{N}(b)$, respectively. Since $G$ is generated by two sets

$$
\left\{a^{x_{a} / t^{\nu_{t}\left(x_{a}\right)}}: \text { prime } t \mid x_{a}\right\} \text { and }\left\{b^{x_{b} / t^{\nu_{t}\left(x_{b}\right)}} \text { : prime } t \mid x_{b}\right\},
$$

if the subgroup $G$ of $(\mathbb{Z} / N \mathbb{Z})^{*}$ generated by $a$ and $b$ is not cyclic, there must exist a common prime divisor $s$ of $x_{a}$ and $x_{b}$ such that the subgroup generated by $a_{s}=$ $a^{x_{a} / s^{\nu_{s}\left(x_{a}\right)}} \bmod N$ and $b_{s}=b^{x_{b} / s^{\nu_{s}\left(x_{b}\right)}} \bmod N$ is not cyclic. Thus, while applying the technique in Lemma 5.5 to each common prime divisor $t$ of $x_{a}$ and $x_{b}$, we can either find a nontrivial divisor of $N$ or conclude that the subgroup $G$ is cyclic. In the latter case, the subgroup $G$ is of order $\operatorname{lcm}\left(x_{a}, x_{b}\right)$, and it is generated by $g=$ $\prod_{\text {prime } t \mid x_{a} x_{b}} g_{t}$, where $g_{t}=a^{x_{a} / \nu_{t}\left(x_{a}\right)}$ if $\nu_{t}\left(x_{a}\right) \geq \nu_{t}\left(x_{b}\right)$; otherwise, $g_{t}=b^{x_{b} / \nu_{t}\left(x_{b}\right)}$.

The overall complexity is

$$
\begin{aligned}
& \sum_{\text {prime } t \mid d} \mathcal{O}\left(\nu_{t}(d)(\log t+\log \log N) \mathrm{M}_{\mathrm{p}}\left(t^{1 / 2}\right) \mathrm{M}(\log N)+\nu_{t}(d)^{2} \log t \mathrm{M}(\log N)\right) \\
= & \mathcal{O}\left(\log d \log \log N \mathrm{M}_{\mathrm{p}}\left(s^{1 / 2}\right) \mathrm{M}(\log N)+\log ^{2} d \mathrm{M}(\log N)\right)
\end{aligned}
$$

bit operations, where $s$ is the largest prime number dividing $d=\operatorname{gcd}\left(x_{a}, x_{b}\right)$. The storage requirement is $\mathcal{O}\left(s^{1 / 2} \log s \log N\right)$ bits.

Note that it is sufficient to know the complete factorization of the greatest common divisor $d$ of $\operatorname{ord}_{N}(a)$ and $\operatorname{ord}_{N}(b)$ to deduce the result of Lemma 5.3. In particular, if $d=1$, the subgroup $G$ is generated by $a b$, and hence, it is cyclic.

To summarize all the procedures described in the proof of Lemma [5.5] and Lemma 5.3 in one procedure, we have the following algorithm denoted as Algorithm 2.

Given inputs $a, b, x_{a}=\operatorname{ord}_{N}(a), x_{b}=\operatorname{ord}_{N}(b)$, and complete factorizations of $x_{a}$ and $x_{b}$, do

(1) Set $\zeta=1$.

(2) If $d:=\operatorname{gcd}\left(x_{a}, x_{b}\right)=1$, go to Step (4).

(3) For each prime divisor $s$ of $d$, do

(3.a) Set $a_{s}=a^{x_{a} / s^{\nu_{s}\left(x_{a}\right)}}$ and $b_{s}=b^{x_{b} / s^{\nu_{s}\left(x_{b}\right)}}$.

(3.b) If $\nu_{s}\left(x_{a}\right) \geq \nu_{s}\left(x_{b}\right)$, set $g=a_{s}^{s^{\nu_{s}\left(x_{a}\right)-1}}$ and $e=\nu_{s}\left(x_{a}\right)$.

Otherwise, set $g=b_{s}^{s^{\nu_{s}\left(x_{b}\right)-1}}$ and $e=\nu_{s}\left(x_{b}\right)$.

(3.c) Set $x=0$.

(3.d) For $i=0$ to $e-1$, do

(i) Set $h_{i}=\left(b_{s} a_{s}^{-x}\right)^{s^{e-(i+1)}} \bmod N$.

(ii) Compute $x_{i} \in[0, s)$ such that $d_{i}=\operatorname{gcd}\left(h_{i}-g^{x_{i}}, N\right)>1$ by applying the technique described in Lemma 3.1

(iii) If $d_{i}<N$, then output this and stop.

(iv) Set $x=x_{0}+x_{1} s+\cdots+x_{i} s^{i}$.

(3.e) Multiply $\zeta$ by $g$.

(4) After multiplying $\zeta$ by $(a b)^{d}$, output this and complete factorization of $\operatorname{lcm}\left(x_{a}, x_{b}\right)$. 
5.5. Remark. We may consider an extension of Pollard's algorithm [15, Theorem 1] to the interval case $[\alpha, \beta]$. That is to say, combining Lemma 3.1 Lemma 5.2. and Lemma 3.2, we can construct another deterministic algorithm computing a nontrivial divisor of $N$ when it has a prime divisor in the interval $[\alpha, \beta]$. Let us briefly explain this algorithm. Let $\gamma$ be a positive real number. Once we fail to find a nontrivial divisor of $N$ by applying Lemma 3.1 and Lemma 5.2 to all integers $2 \leq a \leq A=\left\lceil\beta^{\gamma}\right\rceil$, we know $x_{a}=\operatorname{ord}_{N}(a)$ and $\operatorname{ord}_{N}(a)=\operatorname{ord}_{n}(a)$ for any divisor $n$ of $N$. In this case, as explained in Section 3.2.1, we can find a nontrivial divisor of $N$ by testing if $z H+1 \mid N$ for integers $z$ in $\left[1, C(1 / \gamma)^{1 / \gamma}\right]$, where $H=\operatorname{lcm}\left(x_{2}, \ldots, x_{A}\right)$ and $C$ is the number defined in Lemma 3.2

Note that the described method leads to a deterministic factoring algorithm with complexity $\widetilde{\mathcal{O}}\left(\beta^{\gamma} \delta^{1 / 2}+(1 / \gamma)^{1 / \gamma}\right)$ for any $\gamma>0$. If $\alpha=0$, as in the case of Pollard, or $\beta=O\left(\delta^{k}\right)$ for some $k>0$, the complexity is $\widetilde{\mathcal{O}}\left(\delta^{1 / 2}\right)$, which is satisfactory. However, if $\beta$ grows much faster than $\delta$, this complexity cannot be bounded by $\widetilde{\mathcal{O}}\left(\delta^{1 / 2}\right)$. For example, if $\beta=e^{\delta}$, the complexity is at least $\Omega(\delta)$.

\section{Comparison}

The problem of finding a divisor in an interval can be solved by making use of the seminal works of Coppersmith [5,6]. Using lattice basis reduction algorithms such as LLL [11], Coppersmith presented a method for finding small roots of modular polynomial equations. He used these ideas to obtain an algorithm for finding a prime divisor $p$ given RSA modulus $N=p q$ and an approximation of $p$. Coppersmith originally used a bivariate polynomial method, but we will use a simpler version following the work of Boneh, Durfee, and Howgrave-Graham 4, who used a univariate polynomial method.

Coppersmith's method gives a polynomial time algorithm for finding a divisor in an interval if $\beta-\alpha$ is sufficiently small. More precisely, by letting $p$ be a divisor (not necessarily prime) of $N$ with $p \geq N^{\delta}$, given two numbers $\alpha$ and $\beta$ with $\beta-$ $\alpha \leq N^{\delta^{2}}$, the divisor $p$ can be recovered in polynomial time in $\log N$. However, beyond the (error) bound $N^{\delta^{2}}$, Coppersmith's method is exponential in the size of $(\beta-\alpha) / N^{\delta^{2}}$. This is summarized in the following theorem, which is a reformulation of Coppersmith's results by May [13, Theorem 1].

Theorem 6.1. Let $N$ be an integer of unknown factorization, which has a divisor $M \geq N^{\delta}, 0<\delta \leq 1$, let $f(x)$ be a univariate monic polynomial of degree $d$, and let $c \geq 1$. Then, we can find all solutions $x_{0}$ for the equation

$$
f(x) \equiv 0 \bmod M \quad \text { with } \quad\left|x_{0}\right| \leq c N^{\delta^{2} / d}
$$

in time $\mathcal{O}\left(c d^{5} \log ^{9} N\right)$.

Following Boneh, Durfee, and Howgrave-Graham, we can solve the problem of finding a divisor in an interval. Let us consider the polynomial $f(x)=x+\tilde{p}$, where $\tilde{p}=\lfloor(\alpha+\beta) / 2\rfloor$. If there is a divisor $p \in[\alpha, \beta]$ of $N$, the polynomial $f(x)$ has a solution $x_{0}:=p-\tilde{p} \bmod p$. Since $\left|x_{0}\right| \leq(\beta-\alpha) / 2$, by setting $M=p, N^{\delta}=\alpha$, and $d=1$, the above theorem gives all such solutions $x_{0}$, and hence, all $p \in[\alpha, \beta]$ in time

$$
\mathcal{O}\left(c \log ^{9} N\right), \text { where } c=\max \left\{1,(\beta-\alpha) \cdot 2^{-\frac{\log ^{2} \alpha}{\log N}-1}\right\}
$$




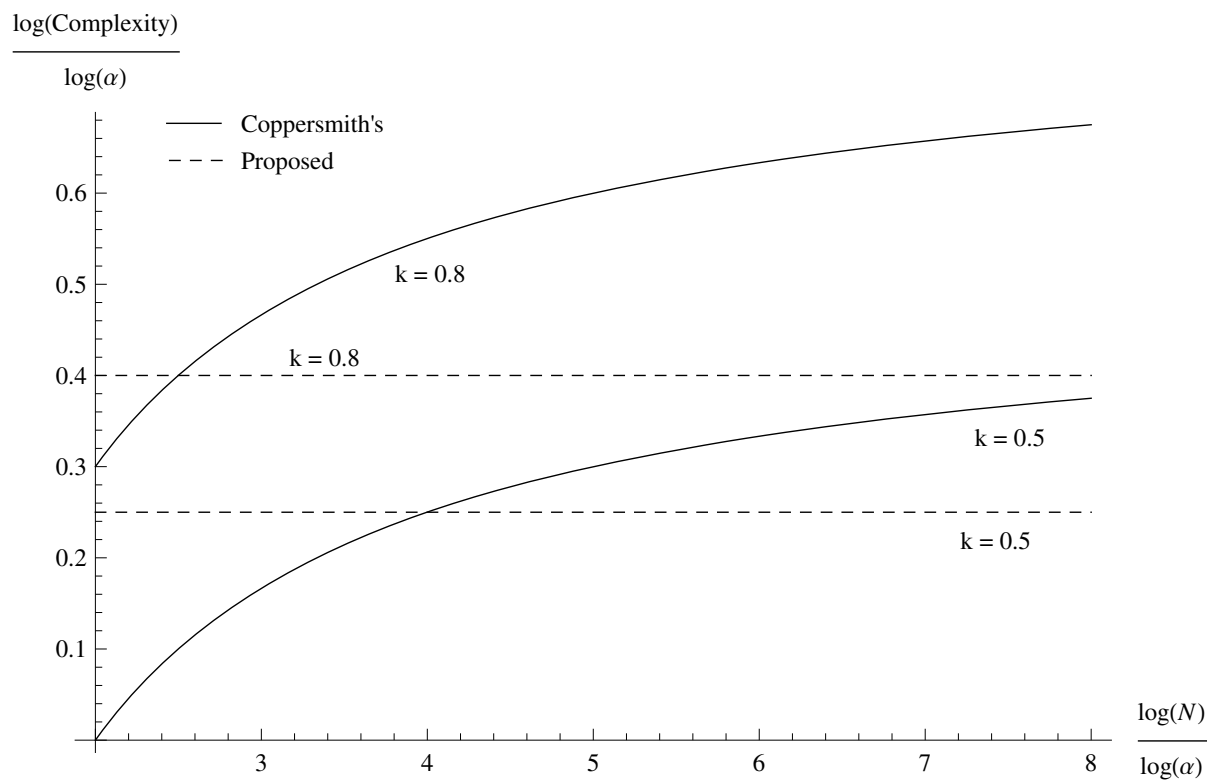

Figure 1. Comparison of complexities excluding logarithmic factors $(k=\log (\beta-\alpha) / \log \alpha)$

If all the solutions $x_{0}$ do not satisfy $\left(x_{0}+\tilde{p}\right) \mid N$, we can conclude that there is no divisor of $N$ in the interval $[\alpha, \beta]$.

Coppersmith's method depends on not only the interval length but also the relative size of the divisor for the target composite number $N$. On the other hand, the proposed algorithms mainly depend on the interval length. This difference makes our method better than Coppersmith's method. Adopting FFT-based multiplication algorithms and disregarding logarithmic factors in (4.1) and (5.1), the running time of the proposed algorithms can be considered as $\mathcal{O}\left((\beta-\alpha)^{1 / 2}\right)$. Comparing it with (6.1), the proposed algorithms are more efficient than Coppersmith's method when

$$
(\beta-\alpha) \cdot 2^{-\frac{\log ^{2} \alpha}{\log N}-1}>(\beta-\alpha)^{1 / 2}
$$

which is equivalent to

$$
\log N>\frac{2 \log ^{2} \alpha}{\log (\beta-\alpha)-2}
$$

Thus, as $\log N$ becomes larger or $\log (\beta-\alpha)$ gets closer to $\log \alpha$, our algorithms are more efficient than Coppersmith's method. This feature is well displayed in Figure 1]

Finally, we remark that our algorithms are effective for moderate sizes of $\log \beta$. If $\log \beta$ is sufficiently large and $\log (\beta-\alpha)$ is not, Lenstra's Elliptic Curve Method [10] will be more efficient. 


\section{Conclusions and Further studies}

We addressed the problem of finding a nontrivial divisor of a given composite integer $N$ when it has a prime divisor in a given interval. We showed that this problem can be solved in around the time of the square root of the interval length, by using a similar amount of storage. The same technique can be applied to devise a probabilistic algorithm for the case of composite divisors, but it is open to design its deterministic version. It would be interesting to develop a low-storage algorithm that finds a divisor in an interval, such as Pollard's rho or kangaroo method [17. used for solving discrete logarithm problems.

\section{REFERENCES}

[1] Manindra Agrawal, Neeraj Kayal, and Nitin Saxena, PRIMES is in P, Ann. of Math. (2) 160 (2004), no. 2, 781-793, DOI 10.4007/annals.2004.160.781. MR.2123939(2006a:11170)

[2] E. Bach, Discrete logarithms and factoring, Technical Report, University of California at Berkely.

[3] Eric Bach, Explicit bounds for primality testing and related problems, Math. Comp. 55 (1990), no. 191, 355-380, DOI 10.2307/2008811. MR1023756 (91m:11096)

[4] Dan Boneh, Glenn Durfee, and Nick Howgrave-Graham, Factoring $N=p^{r} q$ for large $r$, Advances in cryptology - CRYPTO '99 (Santa Barbara, CA), Lecture Notes in Comput. Sci., vol. 1666, Springer, Berlin, 1999, pp. 326-337, DOI 10.1007/3-540-48405-1_21. MR.1729303 (2000i:11188)

[5] Don Coppersmith, Finding a small root of a univariate modular equation, Advances in cryptology_EUROCRYPT '96 (Saragossa, 1996), Lecture Notes in Comput. Sci., vol. 1070, Springer, Berlin, 1996, pp. 155-165, DOI 10.1007/3-540-68339-9_14. MR.1421584|(97h:94008)

[6] Don Coppersmith, Small solutions to polynomial equations, and low exponent RSA vulnerabilities, J. Cryptology 10 (1997), no. 4, 233-260, DOI 10.1007/s001459900030. MR.1476612 (99b:94027)

[7] Joachim von zur Gathen and Jürgen Gerhard, Modern computer algebra, 2nd ed., Cambridge University Press, Cambridge, 2003. MR2001757 (2004g:68202)

[8] G. H. Hardy and E. M. Wright, An introduction to the theory of numbers, 5th ed., The Clarendon Press Oxford University Press, New York, 1979. MR568909 (81i:10002)

[9] Sergei Konyagin and Carl Pomerance, On primes recognizable in deterministic polynomial time, The mathematics of Paul Erdős, I, Algorithms Combin., vol. 13, Springer, Berlin, 1997, pp. 176-198, DOI 10.1007/978-3-642-60408-9_15. MR1425185(98a:11184)

[10] H. W. Lenstra Jr., Factoring integers with elliptic curves, Ann. of Math. (2) 126 (1987), no. 3, 649-673, DOI 10.2307/1971363. MR916721 (89g:11125)

[11] A. K. Lenstra, H. W. Lenstra Jr., and L. Lovász, Factoring polynomials with rational coefficients, Math. Ann. 261 (1982), no. 4, 515-534, DOI 10.1007/BF01457454. MR682664 (84a:12002)

[12] H. W. Lenstra, Jr. and C. Pomerance, Primality testing with Gaussian periods, Manuscript, math.dartmouth.edu/ carlp, 2005.

[13] A. May, Using LLL-reduction for solving RSA and factorization problems: A Survey, LLL+25 Conference in honour of the 25th birthday of the LLL algorithm, 2007.

[14] A. J. Menezes, P. C. van Oorschot, and S. A. Vanstone, Handbook of applied cryptography, CRC Press, 1996.

[15] J. M. Pollard, Theorems on factorization and primality testing, Proc. Cambridge Philos. Soc. 76 (1974), 521-528. MR0354514 (50 \#6992)

[16] J. M. Pollard, A Monte Carlo method for factorization, Nordisk Tidskr. Informationsbehandling (BIT) 15 (1975), no. 3, 331-334. MR0392798 (52 \#13611)

[17] J. M. Pollard, Monte Carlo methods for index computation $(\bmod p)$, Math. Comp. 32 (1978), no. 143, 918-924. MR0491431 (58 \#10684)

[18] R. L. Rivest, A. Shamir, and L. Adleman, A method for obtaining digital signatures and public-key cryptosystems, Comm. ACM 21 (1978), no. 2, 120-126, DOI 10.1145/359340.359342. MR700103(83m:94003) 
[19] A. Schönhage and V. Strassen, Schnelle Multiplikation grosser Zahlen (German, with English summary), Computing (Arch. Elektron. Rechnen) 7 (1971), 281-292. MR0292344|(45 \#1431)

[20] H. C. Williams, A p 1 method of factoring, Math. Comp. 39 (1982), no. 159, 225-234, DOI 10.2307/2007633. MR658227 (83h:10016)

Electronics and Telecommunications Research Institute, P. O. Box 1, Yuseong, DaeJEON, 305-600, KOREA

E-mail address: mkkim@ensec.re.kr

ISaC and Department of Mathematical Sciences, Seoul National University, Seoul 151-747, KOREA

E-mail address: jhcheon@snu.ac.kr 